\title{
Thermodynamic phase transition based on the nonsingular temperature
}

\author{
Myungseok Eune, ${ }^{1, *}$ Yongwan Gim, ${ }^{2, \dagger}$ and Wontae $\mathrm{Kim}^{2, \ddagger}$ \\ ${ }^{1}$ Department of Computer System Engineering, \\ Sangmyung University, Cheonan, 330-720, Republic of Korea \\ ${ }^{2}$ Department of Physics, Sogang University, Seoul 121-742, Republic of Korea
}

(Dated: August 24, 2021)

\begin{abstract}
The Hawking temperature for the Schwarzschild black hole is divergent when the mass of the black hole vanishes; however, the corresponding geometry becomes the Minkowski spacetime whose intrinsic temperature is zero. In connection with this issue, we construct a nonsingular temperature which follows the Hawking temperature for the large black hole, while it vanishes when the black hole is completely evaporated. For the thermodynamic significances of this modified temperature, we calculate thermodynamic quantities and study phase transitions. It turns out that even the small black hole can be stable below a certain temperature, and the hot flat space is always metastable so that it decays into the stable small black hole or the stable large black hole.
\end{abstract}

\footnotetext{
* eunems@smu.ac.kr

$\dagger$ yongwan89@sogang.ac.kr

$\ddagger$ wtkim@sogang.ac.kr
} 


\section{INTRODUCTION}

Bekenstein has suggested that a black hole should have an entropy which is proportional to the area of the horizon [1-3], and Hawking has shown that there is radiation from the black hole through the analysis for the origin of the entropy from the point of view of quantum field theory [4]. The Hawking temperature could be defined generically as $T_{\mathrm{H}}=\hbar \kappa_{H} /(2 \pi)$, where $\kappa_{H}$ is the surface gravity at the horizon. Subsequently, the thermodynamics of the black hole based on this temperature has been one of the most important issues in black hole physics [5-7], so that there have been much intensive study of thermodynamics and phase transitions in various black holes [8-25]. Such phase transitions could be easily read off from the behaviors of the heat capacity and the free energy. In particular, considering the Schwarzschild black hole in a cavity which defines the isothermal surface [7], it was shown that the hot flat space is more probable than the large black hole below a critical temperature, while the large black hole is more probable than the hot flat space above the critical temperature. In fact, the most essential ingredient in the thermodynamics of black holes is to define the black hole temperature such as the Hawking temperature for the Schwarzschild black hole given as $T_{\mathrm{H}}=\hbar /(8 \pi G M)$ from the surface gravity, where $G$ and $M$ are the gravitational constant and the mass of the black hole, respectively. However, it shows that the temperature is proportional to the inverse of the mass, and it is divergent when the mass of the black hole vanishes, although the black hole disappears and its metric becomes the Minkowski spacetime.

The conventional method to resolve the above singular behavior of the Hawking temperature for the Schwarzschild black hole is to introduce the Planck mass as a cutoff $M_{\mathrm{P}}$ in the regime of the generalized uncertainty principle (GUP) [26-32], and then the GUP temper-

ature is obtained as $T_{\mathrm{GUP}}=M /(4 \pi)\left[1 \pm \sqrt{1-M_{\mathrm{P}}^{2} / M^{2}}\right]$ [33]. It produces the well-known Hawking temperature for the large black hole, and it is finite at $M=M_{\mathrm{P}}$. Thanks to this cutoff, the Hawking temperature can be regular as long as $M \geq M_{\mathrm{P}}$; however, it requires the remnant which has a nonvanishing temperature at the order of the Planck mass. Moreover, the stability of the remnant is not warranted since the heat capacity of the remnant approaches negative zero [34]. There have been extensive studies of the thermodynamics of black holes using the GUP temperature, [35-38], and the discussion for the above issues of GUP by deforming the Einstein-Hilbert action which leads to the formation of a zero 
temperature stable remnant [39].

On the other hand, there has been another nontrivial attempt to derive the entropy and temperature which give eventually finite results when the mass of the black hole goes to zero by defining a new temperature based on the argument of the high order of quantum corrections [40, 41]. It turns out that the entropy goes to zero, while the temperature is still divergent when the black hole evaporates completely. Note that one parameter in the temperature is replaced by a mass-dependent one, so that it is possible to make the temperature finite as was shown in Ref. [40]; however, it is still nonvanishing when the mass of the black hole goes to zero. Now, one might wonder how to get the entropy and temperature which follow the well-known ordinary Hawking temperature and entropy and the vanishing temperature and entropy at the end state of the black hole without any remnants.

In this work, we would like to present a nonsingular temperature without resort to the cutoff in the UV region. Of course, the complete quantum gravity beyond the Planck scale is not yet known, but for our purpose, we just demand two boundary conditions - that the temperature should follow the ordinary Hawking temperature for the large black hole and vanish when the mass of the black hole becomes zero. The latter condition is plausible if the end state of evaporation of the black hole naturally becomes the Minkowski spacetime without the remnant. In Sec. II, we start with a somewhat general finite temperature, satisfying the above two boundary conditions. Apart from these conditions, we assume that the entropy of the black hole is positive, and it also vanishes when the mass of the black hole goes to zero. At last, we determine the most simple but regular temperature satisfying the desired asymptotic behaviors among infinite number of ways to reach the Minkowskian spacetime. In Sec. III, in order to exhibit the thermodynamic behaviors of the temperature, the above nonsingular temperature will be localized in the cavity following the work in Ref. [7]. Then, we find that the localized temperature has two extrema, so that there exist two critical masses of $M_{0}$ and $M_{1}$ compared to the single critical mass $M_{1}$ in the standard calculation in the cavity. The conventional thermodynamic calculation showed that the small black hole is always unstable; however, in this case it will be shown that the small black hole can also be stable. Next, in order for studying phase transition based on the newly defined temperature, the free energies will be considered for the hot flat space, the small black hole, and the large black hole, respectively. Then, we find a Hawking-Page-type 
phase transition between the small black hole and the large black hole. Additionally, we find that the hot flat space is always metastable, and it decays into the small black hole or the large black hole. Finally, the conclusion and discussion are given in Sec. IV.

\section{NONSINGULAR TEMPERATURE OF THE BLACK HOLE}

In the Schwarzschild black hole, the Hawking temperature becomes singular at $M \rightarrow 0$, so that it is nontrivial task to describe this region properly by means of the ordinary Hawking temperature. So, let us assume that the Hawking temperature can be modified in such a way that the temperature of the black hole vanishes when the mass of the black hole goes to zero while it follows the behavior of the well-known Hawking temperature for the large black hole. For this purpose, let us write temperature as

$$
T=\frac{1}{8 \pi G M} t(M)
$$

where $t(M)$ should be chosen in order to satisfy the two boundary conditions mentioned earlier and a more or less general expression in terms of polynomial expansion of the mass can be written as

$$
t(M)=\frac{\sum_{i=0}^{n} a_{i}\left(\frac{M}{M_{\mathrm{P}}}\right)^{1+\alpha_{i}}}{\sum_{i=0}^{n} b_{i}\left(\frac{M}{M_{\mathrm{P}}}\right)^{1+\beta_{i}}+C}
$$

where $t \sim M^{1+\alpha_{0}}$ for $M \rightarrow 0$ and $t \sim O(1)$ for $M \rightarrow \infty$, and $\alpha_{i}, \beta_{i}$, and $C$ are positive constants with $\alpha_{n}=\beta_{n}$. Additionally, $\alpha_{i}<\alpha_{j}, \beta_{i}<\beta_{j}$ for $i<j$, and $a_{n}=b_{n}$. Note that the similar ansatz for the temperature appeared with the same spirit in terms of the expansion of integer powers in Ref. [40]; however, any fractional powers are allowed in Eq. (2) without loss of generality. Compactly, the modified temperature for the black hole can be rewritten as

$$
T=\frac{1}{8 \pi G M} \frac{\sum_{i=0}^{n} a_{i}\left(\frac{M}{M_{\mathrm{P}}}\right)^{1+\alpha_{i}}}{\sum_{i=0}^{n} b_{i}\left(\frac{M}{M_{\mathrm{P}}}\right)^{1+\beta_{i}}+C}
$$

Then, the entropy calculated from the first law of thermodynamics,

$$
S=\int \frac{d M}{T},
$$


still respects the Bekenstein-Hawking entropy for the large black hole as $S \sim 4 \pi G M^{2}$. On the other hand, the entropy (4) for the small mass can be calculated as

$$
S=\frac{8 \pi G M_{\mathrm{P}} C}{a_{0}} \int\left(\frac{M_{\mathrm{P}}}{M}\right)^{\alpha_{0}} d M \sim \begin{cases}-\frac{1}{M^{\alpha_{0}-1}} & \text { for } \alpha_{0}>1 \\ \ln M & \text { for } \alpha_{0}=1 \\ M^{1-\alpha_{0}} & \text { for } 0<\alpha_{0}<1\end{cases}
$$

where we neglected the subleading terms. Note that the entropy is negative divergent for $\alpha_{0} \geq 1$, and it vanishes for $0<\alpha_{0}<1$ when $M \rightarrow 0$. As a result, we obtain the additional condition of $0<\alpha_{0}<1$ in order for the positive entropy.

The most simple form of the modified temperature (3) without loss of generality corresponds to $n=0$, which is written as

$$
T=\frac{1}{8 \pi G M}\left[1+\frac{1}{\alpha}\left(\frac{M_{\mathrm{P}}}{M}\right)^{1+\alpha}\right]^{-1},
$$

where we used the relation of $M_{\mathrm{P}}^{2}=G^{-1}, \alpha_{0}=\beta_{0}, a_{0}=b_{0}$, and in particular chose $a_{0} C^{-1}=\alpha_{0}$ in order to make the temperature a maximum at $M=M_{\mathrm{P}}$. After calculations, $\alpha_{0}$ was replaced by $\alpha$ for simplicity, so that $0<\alpha<1$ in Eq. (6). The behavior of the temperature (6) is illustrated in Fig. 1 which shows the modified temperature following the Hawking temperature asymptotically and eventually vanishing when the mass of the black hole goes to zero. So, eventually, there is no remnant after complete evaporation of the black hole, and the corresponding geometry becomes the Minkowski spacetime. It is compatible with the fact that the Minkowski spacetime has no intrinsic temperature.

By using Eq. (4), the entropy corresponding to the modified temperature (6) is calculated as

$$
S=4 \pi G M^{2}+\frac{8 \pi}{\alpha(1-\alpha)}\left(\frac{M}{M_{\mathrm{P}}}\right)^{1-\alpha}
$$

where the entropy vanishes for $M \rightarrow 0$. It goes to the Bekenstein-Hawking's area law of the entropy for the large black hole. As a result, the temperature and entropy vanish at the end state of the black hole. The difference from the previous works $[40,41]$ comes from the fact that even the temperature can be zero when the mass of the black hole goes to zero if we use the fractional power of expansion in the temperature expression (6). 


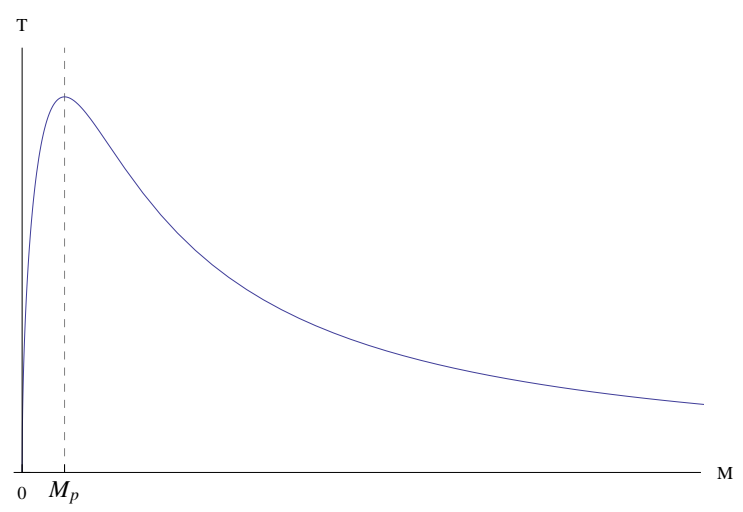

FIG. 1. The modified temperature is plotted by setting the constants as $M_{\mathrm{P}}=1$ and $\alpha=1 / 2$ in Eq. (6). The temperature follows the Hawking temperature asymptotically and vanishes at $M \rightarrow 0$. The maximum temperature appears at the Planck mass.

\section{THERMODYNAMIC QUANTITIES AND PHASE TRANSITION}

In connection with the modified temperature (6), we are going to calculate the relevant thermodynamic quantities which will be employed in order to investigate thermodynamic phase transitions. Let us first consider the cavity as a boundary with a radius $r$ to study quasilocal thermodynamics along the line of the procedure in Ref. [7]. Then, the local temperature measured at the boundary is given as

$$
\begin{aligned}
T_{\mathrm{loc}} & =\frac{T}{\sqrt{1-2 G M / r}} \\
& =\frac{1}{8 \pi G M \sqrt{1-2 G M / r}}\left[1+\frac{1}{\alpha}\left(\frac{M_{\mathrm{P}}}{M}\right)^{1+\alpha}\right]^{-1},
\end{aligned}
$$

which is shown in Fig. 2(a). It shows that there are two extrema: one is the local maximum at $M=M_{0}$ and the other is the local minimum at $M=M_{1}$. Let us now define the large black hole where $M>M_{1}$ and the small black hole where $M<M_{1}$ for convenience. From Fig. 2(a), it can be shown that there is one small black hole for $0<T<T_{0}$, two small black holes and one large black hole $T_{0}<T<T_{1}$, and one large black hole for $T>T_{1}$, respectively. The similar black hole states can be found in the noncommutative black hole [42-45] and the Horava-Lifshitz black hole [46-48]; however, the remnant associated with the parameter which characterizes the model was required.

Note that the entropy (7) is independent of the size of the cavity in quasilocal thermo- 


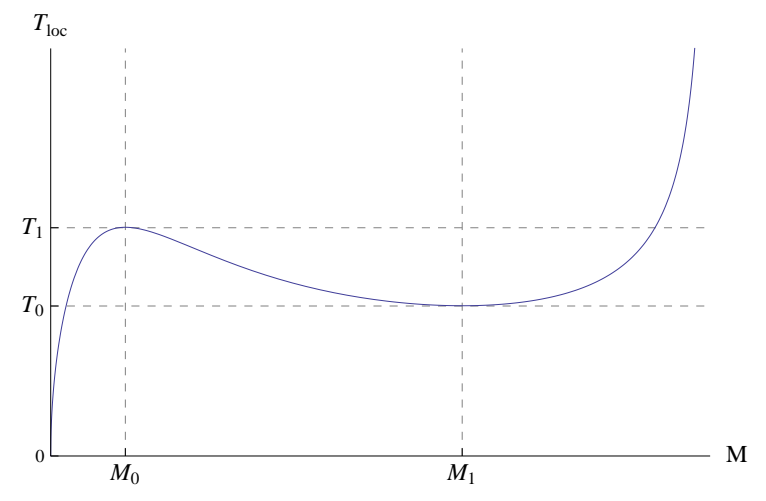

(a)local temperature

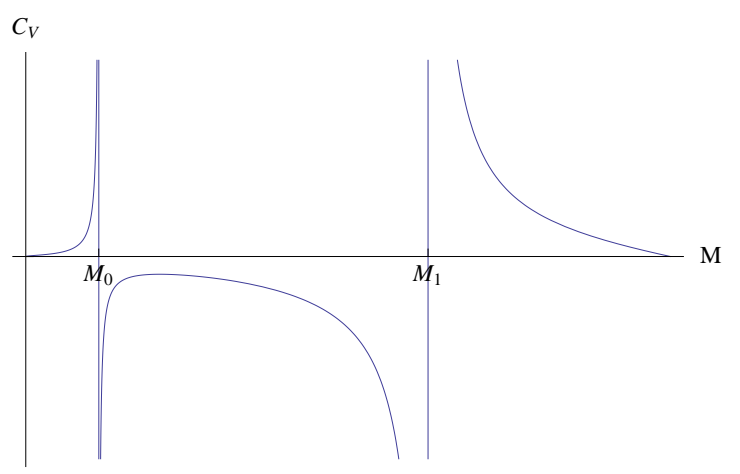

(b)heat capacity

FIG. 2. The local temperature (a) and the heat capacity (b) are plotted by setting the constants as $G=1, \alpha=1 / 2$, and $r=10$, respectively. Figure (a) shows that the number of states of the black hole is subject to the temperature. In Fig. (b), the large black hole in $M>M_{1}$ is always stable, but the small black hole is stable for $M<M_{0}$ whereas it is unstable for $M_{0}<M<M_{1}$.

dynamics [49] since the degrees of freedom of the black hole should be independent of the observer located at the radial distance $r$ [50]. Applying the first law of thermodynamics, the total thermodynamic internal energy within the boundary $r$ is obtained as

$$
\begin{aligned}
E_{\mathrm{loc}} & =\int_{0}^{M} T_{\mathrm{loc}} d S \\
& =\frac{r}{G}-\frac{r}{G} \sqrt{1-\frac{2 G M}{r}},
\end{aligned}
$$

which is the same with the energy obtained from the conventional Hawking temperature [7, 51]. Using Eq. (9), the heat capacity at fixed $r$ is calculated as

$$
\begin{aligned}
C_{V} & =\left(\frac{\partial E_{\mathrm{loc}}}{\partial T_{\mathrm{loc}}}\right)_{r} \\
& =\frac{8 \pi G M^{2}\left(1-\frac{2 G M}{r}\right)\left(1+\frac{1}{\alpha}\left(\frac{M_{\mathrm{P}}}{M}\right)^{1+\alpha}\right)}{\left(\frac{M_{\mathrm{P}}}{M}\right)^{1+\alpha}\left(1-\frac{2 G M}{r}+\frac{1}{\alpha} \frac{G M}{r}\right)-1+\frac{3 G M}{r}} .
\end{aligned}
$$

As seen from Fig. 2(b), the large black hole in $M>M_{1}$ is stable since the heat capacity is positive, which is the same with the ordinary behavior of the heat capacity in the Schwarzschild black hole. However, the small black hole can be either stable or unstable depending on the size of the black hole, so that it is stable for $M<M_{0}$ and unstable for $M_{0}<M<M_{1}$. After all, it turns out that the small black hole for $M<M_{0}$ can be nucleated like the large black hole, which makes the phase transition nontrivial. 


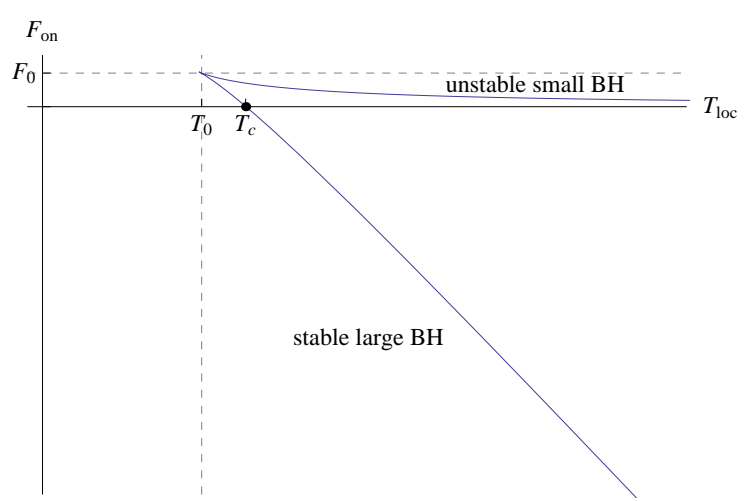

(a) Free energy based on the Hawking temperature

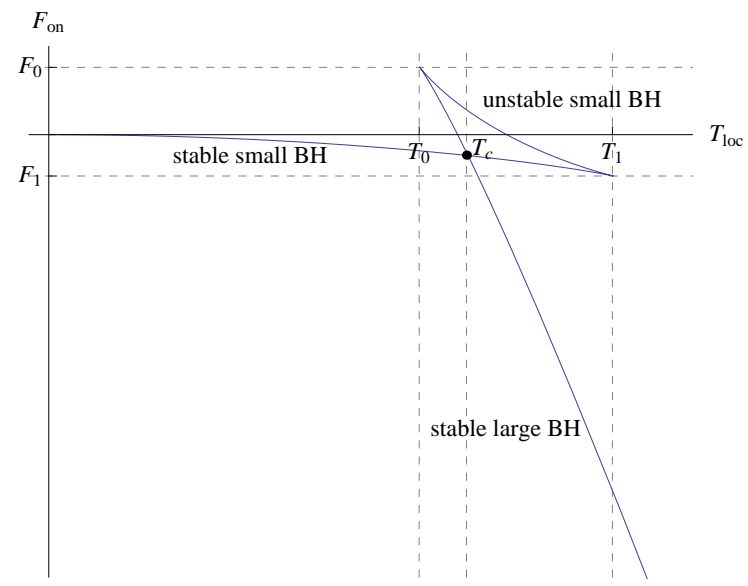

(b) Free energy based on the modified temperature

FIG. 3. The ordinary free energy and the modified free energy are plotted, respectively. The constants are chosen as $G=1, \alpha=1 / 2$, and $r=10$. The phase transition appears between the hot flat space and the stable large black hole in Fig. 3(a), while it happens between the stable small black hole and the stable large black hole in Fig. 3(b). $T_{c}$ is a critical temperature and the other temperatures in Fig. 3(b) were defined in Fig. 2(a).

Next, let us calculate the free energy of the black hole in order to study phase transition between the black holes and the hot flat space. Using Eqs. (7), (8), and (9), the free energy of the black hole is easily obtained as

$$
\begin{aligned}
F_{\mathrm{on}}^{\mathrm{bh}} & =E_{\mathrm{loc}}-T_{\mathrm{loc}} S \\
& =\frac{r}{G}-\frac{r}{G} \sqrt{1-\frac{2 G M}{r}}-\frac{4 \pi G M^{2}+\frac{8 \pi G M_{\mathrm{P}}^{2}}{\alpha(1-\alpha)}\left(\frac{M}{M_{\mathrm{P}}}\right)^{1-\alpha}}{8 \pi G M \sqrt{1-\frac{2 G M}{r}}\left(1+\frac{1}{\alpha}\left(\frac{M_{\mathrm{P}}}{M}\right)^{1+\alpha}\right)} .
\end{aligned}
$$

Note that for the limit of $M_{\mathrm{P}} / M \rightarrow 0$, Eq. (11) is reduced to the well-known free energy of the black hole in the conventional thermodynamics of the Schwarzschild black hole [7], and the free energy of the hot flat space also becomes zero, $F_{\text {on }}^{\text {hfs }}=0$. In this case, as seen from Fig. 3(a), the large black hole is more probable than the hot flat space above the critical temperature $T_{c}$, and the hot flat space is more probable below the critical temperature $T_{c}$.

We are now in a position to discuss the free energy (11) explicitly. The behavior of the stable large black hole in Fig. 3(b) is essentially the same as that of the conventional one in Fig. 3(a). The difference comes from the behavior of the small black hole since the unstable 
small black hole in Fig. 3(b) starts with the positive free energy at $T_{0}$ but is terminated with the negative free energy at $T_{1}$. Note that the free energy of the unstable small black hole is always positive in any temperature in Fig. 3(a). Moreover, the free energy of the newly defined stable small black hole in Fig. 3(b) is always negative in $T<T_{1}$, and the free energy of the hot flat space is still $F_{\mathrm{on}}^{\mathrm{hfs}}=0$ since the free energy (11) vanishes for $M \rightarrow 0$, so that the free energy of the stable small black hole is lower than the free energy of the hot flat space. So, the hot flat space can collapse not only to the stable large black hole for $T>T_{1}$ but also to the stable small black hole for $T<T_{0}$. In particular, as for $T_{0}<T<T_{1}$, the Hawking-Page-type critical phenomenon can appear between the stable small black hole and the stable large black hole, which is compared to the conventional phase transition between the hot flat space and the stable large black hole as seen from Fig. 3(a). Thus, the stable small black hole is more probable for $T_{0}<T<T_{c}$ and the stable large black hole is more probable for $T_{c}<T<T_{1}$. Additionally, the hot flat space in $T_{0}<T<T_{1}$ decays into the stable small black hole or the stable large black hole eventually.

\section{CONCLUSION AND DISCUSSION}

When the Schwarzschild black hole completely evaporates, the metric describing the system becomes the Minkowski spacetime, although the Hawking temperature is divergent; however, the Minkowski spacetime does not have its own intrinsic temperature since there is no event horizon. In this work, we considered the modified temperature for the Schwarzschild black hole by requiring two conditions: that it should follow the Hawking temperature for the large black hole and vanish when the mass of the black hole goes to zero. The modified temperature was designed in order to show the regular behavior of the temperature of the black hole without encountering any singularity when $M \rightarrow 0$. On the other hand, the several constants in the temperature (6) imply that there are an infinite number of ways for the modified temperature to reach the origin; however, we fixed the constants in such a way that the maximum value of the temperature appears at the Planck scale and fortunately only a single constant remains unfixed. So the simplest form of the temperature could be obtained, but it shares the above two conditions. By the use of this temperature, thermodynamic quantities were calculated in order to study thermodynamic stability and phase transition

of the black hole along the well-established procedure. It turns out that there is a single 
state of the stable small black hole for $T<T_{0}$ or the stable large black hole for $T>T_{1}$. For $T_{0}<T<T_{1}$, there are three black hole states which consist of the two small black holes and one large black hole. Apart from the existence of the stable small black hole, the most interesting thing to be distinguished from the standard thermodynamics is that the flat space is no longer a stable state thermodynamically in any temperature since it should always decay into the stable small black hole or the stable large black hole, so that the final state becomes a black hole state.

Finally, the method for the present regular temperature requires an explanation about the limitations of the present approach since we assumed a certain modification of the Hawking temperature as a function of the black hole mass but did not discuss the origin of such a modification. First of all, the temperature (6) derived from the polynomial expansion of the black hole mass is, indeed, not unique even in spite of the plausibility of reproducing the conventional Hawking temperature; for example, another type of temperature such as $T=1 /(8 \pi G M)\left(1-e^{-k\left(M / M_{p}\right)^{1+\alpha}}\right)$, where $k$ is an arbitrary positive constant also satisfies the two boundary conditions mentioned in Sec. II. To fix the physically meaningful temperature uniquely and figure out what happens at the end state of evaporation of the black hole, the complete theory of quantum gravity covering the trans-Planckian regime should be defined. The second limitation of our approach is that we employed the classical metric for the local Tolman temperature as seen from Eq. (8), which is a temporary expedient. In particular, one can expect that such a modified temperature (6) comes from a change in the spacetime geometry, so that the local temperature (8) changes accordingly. Note that modifications in the geometry could have a nontrivial effect on our analysis and an impact on the physics of small black holes. This was noticed in the GUP regime, which yields the corresponding GUP temperature, and the classical geometry should be changed according to the modification of the uncertainty relation [39]. Based on this fact, using the one-to-one correspondence between the GUP and the GUP temperature, a corresponding modified uncertainty relation, which is written as $\Delta x \Delta p+\left(2 \ell_{p} / \alpha\right)\left(2 \ell_{p} / \Delta x\right)^{\alpha} \Delta p \geq 1$, can also be derived straightforwardly from Eq. (6). This modified uncertainty relation will modify the classical geometry similar to the result in Ref. [39] so that the local temperature will be changed somehow near the horizon and, consequently, the thermodynamic behaviors of small black holes may be different from the the present results. This deserves further study, which we hope will appear in the future. 


\section{ACKNOWLEDGMENTS}

We would like to thank E. J. Son for exciting discussions. W.K. was supported by the National Research Foundation of Korea(NRF) Grant No. 2014R1A2A1A11049571, funded by the Korean government(MSIP).

[1] J. D. Bekenstein, Black holes and the second law, Lett. Nuovo Cim. 4 (1972) 737-740.

[2] J. D. Bekenstein, Black holes and entropy, Phys. Rev. D 7 (1973) 2333-2346.

[3] J. D. Bekenstein, Generalized second law of thermodynamics in black hole physics, Phys. Rev. D 9 (1974) 3292-3300.

[4] S. Hawking, Particle Creation by Black Holes, Commun. Math. Phys. 43 (1975) 199-220.

[5] D. Gross, M. Perry, and L. Yaffe, Instability of Flat Space at Finite Temperature, Phys. Rev. D 25 (1982) 330-355.

[6] S. Hawking and D. N. Page, Thermodynamics of Black Holes in anti-De Sitter Space, Commun. Math. Phys. 87 (1983) 577.

[7] J. York, James W., Black hole thermodynamics and the Euclidean Einstein action, Phys. Rev. D 33 (1986) 2092-2099.

[8] A. Medved and G. Kunstatter, Hamiltonian thermodynamics of charged black holes, Phys.Rev. D59 (1999) 104005, [hep-th/9811052].

[9] R.-G. Cai, Gauss-Bonnet black holes in AdS spaces, Phys. Rev. D 65 (2002) 084014, [hep-th/0109133].

[10] R.-G. Cai, L.-M. Cao, and Y.-W. Sun, Hawking-Page Phase Transition of black Dp-branes and R-charged black holes with an IR Cutoff, JHEP 0711 (2007) 039, [arXiv:0709.3568].

[11] R.-G. Cai, S. P. Kim, and B. Wang, Ricci flat black holes and Hawking-Page phase transition in Gauss-Bonnet gravity and dilaton gravity, Phys. Rev. D 76 (2007) 024011, [arXiv:0705.2469].

[12] G. Clement, J. Fabris, and G. Marques, Hawking radiation of linear dilaton black holes, Phys.Lett. B651 (2007) 54-57, [arXiv:0704.0399].

[13] R. Banerjee and B. R. Majhi, Quantum Tunneling Beyond Semiclassical Approximation, JHEP 0806 (2008) 095, [arXiv:0805.2220]. 
[14] W. Kim and E. J. Son, Thermodynamics of warped AdS(3) black hole in the brick wall method, Phys.Lett. B673 (2009) 90-94, [arXiv:0812.0876].

[15] R.-G. Cai, L.-M. Cao, and N. Ohta, Black Holes in Gravity with Conformal Anomaly and Logarithmic Term in Black Hole Entropy, JHEP 1004 (2010) 082, [arXiv:0911.4379].

[16] Y. S. Myung, Lifshitz black holes in the Horava-Lifshitz gravity, Phys. Lett. B 690 (2010) 534-540, [arXiv:1002.4448].

[17] R. Banerjee, S. Ghosh, and D. Roychowdhury, New type of phase transition in Reissner Nordstrom - AdS black hole and its thermodynamic geometry, Phys.Lett. B696 (2011) 156-162, [arXiv:1008.2644].

[18] R. Banerjee and D. Roychowdhury, Thermodynamics of phase transition in higher dimensional AdS black holes, JHEP 1111 (2011) 004, [arXiv:1109.2433].

[19] D. F. Jardim, M. E. Rodrigues, and M. Houndjo, Thermodynamics of phantom Reissner-Nordstrom-AdS black hole, Eur.Phys.J.Plus 127 (2012) 123, [arXiv:1202.2830].

[20] M. E. Rodrigues, D. F. Jardim, and S. J. Houndjo, Thermodynamics of black plane solution, Gen.Rel.Grav. 45 (2013) 2309-2323, [arXiv:1205.3481].

[21] M. Eune, W. Kim, and S.-H. Yi, Hawking-Page phase transition in BTZ black hole revisited, JHEP 1303 (2013) 020, [arXiv:1301.0395].

[22] E. J. Son and W. Kim, Two critical phenomena in the exactly soluble quantized Schwarzschild black hole, JHEP 1303 (2013) 060, [arXiv:1212.2307].

[23] T. S. Bir and V. G. Czinner, A q-parameter bound for particle spectra based on black hole thermodynamics with Rnyi entropy, Phys.Lett. B726 (2013) 861-865, [arXiv:1309.4261].

[24] Y. Gim and W. Kim, Thermodynamic phase transition in the rainbow Schwarzschild black hole, JCAP 1410 (2014) 003, [arXiv:1406.6475].

[25] Y. Gim, W. Kim, and S.-H. Yi, The first law of thermodynamics in Lifshitz black holes revisited, JHEP 1407 (2014) 002, [arXiv:1403.4704].

[26] T. Padmanabhan, Limitations on the Operational Definition of Space-time Events and Quantum Gravity, Class.Quant.Grav. 4 (1987) L107-L113.

[27] K. Konishi, G. Paffuti, and P. Provero, Minimum Physical Length and the Generalized Uncertainty Principle in String Theory, Phys.Lett. B234 (1990) 276.

[28] M. Maggiore, The Algebraic structure of the generalized uncertainty principle, Phys.Lett. B319 (1993) 83-86, [hep-th/9309034]. 
[29] M. Maggiore, Quantum groups, gravity and the generalized uncertainty principle, Phys.Rev. D49 (1994) 5182-5187, [hep-th/9305163].

[30] L. J. Garay, Quantum gravity and minimum length, Int.J.Mod.Phys. A10 (1995) 145-166, [gr-qc/9403008].

[31] A. Kempf, G. Mangano, and R. B. Mann, Hilbert space representation of the minimal length uncertainty relation, Phys.Rev. D52 (1995) 1108-1118, [hep-th/9412167].

[32] L. N. Chang, D. Minic, N. Okamura, and T. Takeuchi, The Effect of the minimal length uncertainty relation on the density of states and the cosmological constant problem, Phys.Rev. D65 (2002) 125028, [hep-th/0201017].

[33] R. J. Adler, P. Chen, and D. I. Santiago, The Generalized uncertainty principle and black hole remnants, Gen.Rel.Grav. 33 (2001) 2101-2108, [gr-qc/0106080].

[34] W. Kim, E. J. Son, and M. Yoon, Thermodynamics of a black hole based on a generalized uncertainty principle, JHEP 0801 (2008) 035, [arXiv:0711.0786].

[35] P. S. Custodio and J. Horvath, The Generalized uncertainty principle, entropy bounds and black hole (non)evaporation in a thermal bath, Class.Quant.Grav. 20 (2003) L197-L203, [gr-qc/0305022].

[36] A. Medved and E. C. Vagenas, When conceptual worlds collide: The GUP and the BH entropy, Phys. Rev. D 70 (2004) 124021, [hep-th/0411022].

[37] Y. S. Myung, Y.-W. Kim, and Y.-J. Park, Black hole thermodynamics with generalized uncertainty principle, Phys.Lett. B645 (2007) 393-397, [gr-qc/0609031].

[38] W. Kim and J. J. Oh, Determining the minimal length scale of the generalized uncertainty principle from the entropy-area relationship, JHEP 0801 (2008) 034, [arXiv:0709.0581].

[39] M. Isi, J. Mureika, and P. Nicolini, Self-Completeness and the Generalized Uncertainty Principle, JHEP 1311 (2013) 139, [arXiv:1310.8153].

[40] D. Singleton, E. C. Vagenas, T. Zhu, and J.-R. Ren, Insights and possible resolution to the information loss paradox via the tunneling picture, JHEP 1008 (2010) 089, [arXiv:1005.3778].

[41] D. Singleton, E. C. Vagenas, and T. Zhu, Self-similarity, conservation of entropy/bits and the black hole information puzzle, JHEP 1405 (2014) 074, [arXiv:1311.2015].

[42] P. Nicolini, A. Smailagic, and E. Spallucci, Noncommutative geometry inspired Schwarzschild black hole, Phys.Lett. B632 (2006) 547-551, [gr-qc/0510112]. 
[43] Y. S. Myung, Y.-W. Kim, and Y.-J. Park, Thermodynamics and evaporation of the noncommutative black hole, JHEP 0702 (2007) 012, [gr-qc/0611130].

[44] P. Nicolini, Noncommutative Black Holes, The Final Appeal To Quantum Gravity: A Review, Int.J.Mod.Phys. A24 (2009) 1229-1308, [arXiv:0807.1939].

[45] W. Kim, E. J. Son, and M. Yoon, Thermodynamic similarity between the noncommutative Schwarzschild black hole and the Reissner-Nordstrom black hole, JHEP 0804 (2008) 042, [arXiv:0802.1757].

[46] R.-G. Cai, L.-M. Cao, and N. Ohta, Thermodynamics of Black Holes in Horava-Lifshitz Gravity, Phys.Lett. B679 (2009) 504-509, [arXiv:0905.0751].

[47] Y. S. Myung and Y.-W. Kim, Thermodynamics of Horava-Lifshitz black holes, Eur.Phys.J. C68 (2010) 265-270, [arXiv:0905.0179].

[48] M. Eune, B. Gwak, and W. Kim, Local thermodynamics of KS black hole, Phys.Lett. B718 (2013) 1505-1509, [arXiv:1209.4698].

[49] J. D. Brown, J. Creighton, and R. B. Mann, Temperature, energy and heat capacity of asymptotically anti-de Sitter black holes, Phys.Rev. D50 (1994) 6394-6403, [gr-qc/9405007].

[50] W. Kim, S. Kulkarni, and S.-H. Yi, Quasilocal Conserved Charges in a Covariant Theory of Gravity, Phys.Rev.Lett. 111 (2013), no. 8 081101, [arXiv:1306.2138].

[51] B. F. Whiting and J. York, James W., Action Principle and Partition Function for the Gravitational Field in Black Hole Topologies, Phys.Rev.Lett. 61 (1988) 1336. 